\title{
Electron Energy Distribution of a Current-Free Double Layer: Druyvesteyn Theory and Experiments
}

\author{
Kazunori Takahashi, ${ }^{1,2, *}$ Christine Charles, ${ }^{1}$ Rod W. Boswell, ${ }^{1}$ and Tamiya Fujiwara ${ }^{2}$ \\ ${ }^{1}$ Space Plasma, Power and Propulsion Group, Research School of Physics and Engineering, The Australian National University, \\ Canberra ACT 0200, Australia \\ ${ }^{2}$ Department of Electrical Engineering and Computer Science, Iwate University, Morioka 020-8551, Japan
}

(Received 7 April 2011; published 11 July 2011)

\begin{abstract}
Electron energy distributions upstream of a current-free double layer (CFDL) contained in a lowcollisional plasma are modeled and compared with experimental results. The experimentally observed electron energy probability functions (EEPFs) with a depleted tail above the energy corresponding to the potential drop of the CFDL can be well approximated by a Druyvesteyn distribution function. Theoretical effective electron temperatures for the Druyvesteyn distribution are in good agreement with the values obtained from the experimental EEPFs over the range of pressure where the CFDL is observed.
\end{abstract}

PACS numbers: $52.30 .-\mathrm{q}$

Electric fields of electric double layers (DLs), which spontaneously form in plasmas, accelerate or trap the charged particles and initial investigations of laboratory DLs focused on the current-driven DL (CDDL) [1-3]. The theoretical solution of the CDDL was derived assuming two counterstreaming plasmas with a resultant net current across the DL, Maxwellian distribution functions, and a collisionless plasma [4,5]. The position and potential drop of the CDDLs strongly depend on the ion and electron velocity or energy distribution functions $[6,7]$.

Subsequent to the theoretical prediction of a new category of DLs [8], i.e., a current-free DL (CFDL), experiments on the current-free mode were successfully demonstrated $[9,10]$. Hairapetian and Stenzel observed the formation of a stationary CFDL when a collisionless two-electron-temperature plasma with an energetic tail of electrons over $80 \mathrm{eV}$ expands into a vacuum; the ions upstream of the DL are accelerated to supersonic velocities by the potential drop [10]. According to a recent theory on this CFDL, its solution requires the condition that the temperature of the energetic-tail electrons is higher by about 10 times that of the low-energy cold electrons [11].

More recently, another CFDL has been described in a number of experiments involving low-collisional, magnetically expanding, inductive- or wave-mode discharges [12]. The rapid potential change of this "low-collisional" CFDL near the expanding magnetic field (with an associated acceleration of the ions by the DL electric field) is now being employed in the development of a plasma thruster, for studies on plasma instabilities [13] and for solar plasma physics [14]. The ion energy distribution functions in the "Hairapetian and Stenzel" CFDL in vacuum and in the low-collisional CFDL are not dissimilar. The latter CFDL has been theoretically investigated: one proposed model required an electron beam accelerated by the DL from the downstream plasma [15], and another model followed the classical sheath theory for Boltzmann electrons [16].
However, a typical electron energy distribution measured about $8 \mathrm{~cm}$ upstream of this CFDL [17], shown in Fig. 1 as open circles, is significantly different from the assumptions of Refs. $[15,16]$. The electron energy probability function (EEPF) shows a depleted tail at the break energy $\varepsilon_{\text {break }}$ corresponding to the DL potential drop as indicated by the vertical arrow in Fig. 1, instead of an electron beam superposed on a Maxwellian distribution. By comparing the EEPFs upstream and downstream of the CFDL, it has been suggested that the depleted, energetic-tail electrons overcome the potential drop of the DL and neutralize the ion beam accelerated by the DL. Hence, it appears that the low-collisional CFDL is sustained by only a single plasma source, and does not require the counterstreaming plasmas and the two-electron-temperature population with the

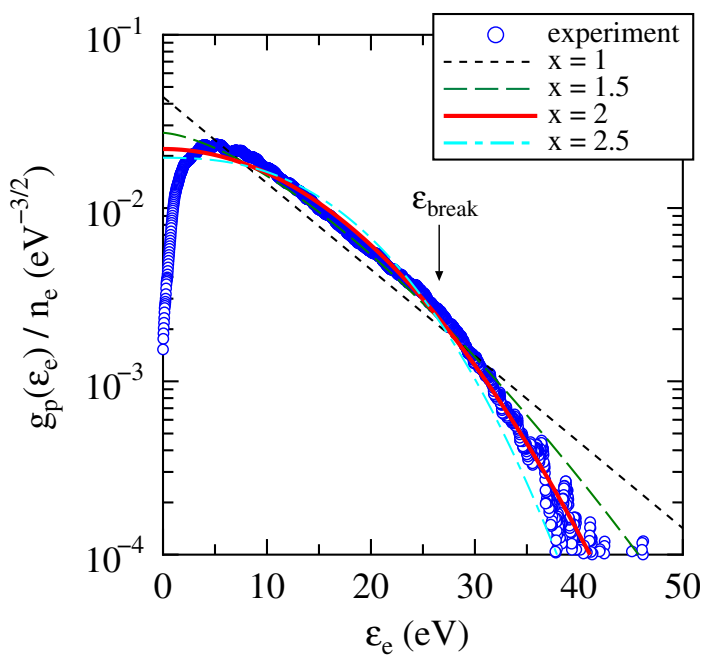

FIG. 1 (color online). Normalized EEPF measured at $8 \mathrm{~cm}$ upstream of the CFDL for 0.3 mTorr of argon in the Chi-Kung reactor (open circles), and the theoretical EEPFs for various values of $x$ with the same effective temperature as in the experiment $\left(T_{\text {eff }} \sim 8.5 \mathrm{eV}\right)$. 
energetic tail as measured in the Hairapetian and Stenzel CFDL. Determining the electron energy distribution function associated with a DL is essential for the understanding of the underlying physics, of the fundamental DL characteristics (position and potential drop), and consequently of its classification.

In this Letter, the electron energy distribution upstream of the low-collisional CFDL is analytically modeled: the EEPFs with the depleted tail are approximately fitted by a Druyvesteyn distribution and the effective electron temperature is analytically obtained. The theoretical results are successfully compared with experimental results obtained for two system geometries and two operating gases.

Although the electron energy distribution can be rigorously obtained by a direct solution of the Boltzmann equation [18], the balance equation between volume ionization and surface loss of the charged particles is presently used as it is a simple and powerful method for determining the electron temperature [19]. In addition, it has been extended to generalized electron energy distributions [20]; no comparison with experiments has yet been reported. The particle balance equation for cylindrical plasmas can be expressed as [19]

$$
K_{i z} n_{g} \pi R^{2} l=u_{B}\left(2 \pi R^{2} h_{l}+2 \pi R l h_{R}\right),
$$

where $K_{i z}, n_{g}, R, l, u_{B}$ are the ionization rate constant, the gas density, the plasma radius, the plasma length, and the Bohm velocity, respectively. $h_{l}$ and $h_{R}$ are the axial and radial center-to-edge density ratios, which are assumed to follow the low-pressure diffusion theory with $h_{l}=$ $0.86\left(3+l / 2 \lambda_{i}\right)^{-1 / 2}$ and $h_{R}=0.8\left(4+R / \lambda_{i}\right)^{-1 / 2}$, where $\lambda_{i}$ is the ion-neutral mean free path. The generalized $\mathrm{EEPF} g_{p}\left(\varepsilon_{e}\right)$ and the distribution function (EEDF) $g_{e}\left(\varepsilon_{e}\right)$ can be expressed as

$$
\begin{gathered}
g_{p}\left(\varepsilon_{e}\right)=g_{x} \frac{n_{e}}{T_{\mathrm{eff}}^{3 / 2}} \exp \left[-C_{x}\left(\frac{\varepsilon_{e}}{T_{\mathrm{eff}}}\right)^{x}\right], \\
g_{e}\left(\varepsilon_{e}\right)=\varepsilon_{e}^{1 / 2} g_{p}\left(\varepsilon_{e}\right),
\end{gathered}
$$

where $\varepsilon_{e}$ and $T_{\text {eff }}$ are the energy of electrons and the effective electron temperature in units of volts, and $x$ is a parameter giving the shape of the EEPF and EEDF; $x=1$ and $x=2$ correspond to Maxwellian and Druyvesteyn distribution functions, respectively. $g_{x}$ and $C_{x}$ are constants derived from the relations between the EEDF and the density $n_{e}$, and between the EEDF and the effective electron temperature $T_{\text {eff }}$, respectively. They can be expressed as $g_{x}=(2 / 3)^{3 / 2} x\left[\Gamma\left(\xi_{1}\right)\right]^{-5 / 2}\left[\Gamma\left(\xi_{2}\right)\right]^{3 / 2}$ and $C_{x}=$ $(2 / 3)^{x}\left[\Gamma\left(\xi_{2}\right) / \Gamma\left(\xi_{1}\right)\right]^{x}$, where $\xi_{1}=3 /(2 x), \quad \xi_{2}=5 /(2 x)$, and $\Gamma(\xi)$ is the Gamma function defined as

$$
\Gamma(\xi)=\int_{0}^{\infty} t^{\xi-1} \exp (-t) d t .
$$

The shape of the EEPF and EEDF, i.e., the value of $x$, affects $u_{B}$ and $K_{i z}$ in Eq. (1). Amemiya [21] reported the sheath formation criterion for non-Maxwellian EEPFs and the generalized Bohm velocity can be derived as

$$
\frac{e}{M} \frac{1}{u_{B}^{2}}=\frac{1}{2 n_{e}} \int_{0}^{\infty} \frac{g_{p}\left(\varepsilon_{e}\right)}{\varepsilon_{e}^{1 / 2}} d \varepsilon_{e},
$$

where $M$ is the ion mass. Substituting the generalized EEPF of Eq. (2) into Eq. (5), the Bohm velocity is

$$
u_{B}=\left(\frac{3 e T_{\text {eff }}}{M}\right)^{1 / 2} \frac{\Gamma\left(\xi_{1}\right)}{\left[\Gamma\left(\xi_{2}\right) \Gamma\left(\xi_{3}\right)\right]^{1 / 2}},
$$

where $\xi_{3}=1 /(2 x)$. The ionization rate constant $K_{i z}$ is analytically derived by using the Thomson cross section: $\sigma_{i z}\left(\varepsilon_{e}\right)=\alpha \pi\left(e / 4 \pi \epsilon_{0}\right)^{2}\left(1 / \varepsilon_{e}\right)\left(1 / \varepsilon_{i z}-1 / \varepsilon_{e}\right)$ for $\varepsilon_{e} \geq$ $\varepsilon_{i z}$ and 0 for $\varepsilon_{e} \leq \varepsilon_{i z}$ [19], where $\epsilon_{0}$ and $\varepsilon_{i z}$ are the permittivity of free space and the ionization energy, respectively. $\alpha=6$ from Ref. [19] is a coefficient used to fit the approximate equation with the actual cross section. As the ionization rate constant $K_{i z}$ can be expressed as Eq. (7) below, it can be analytically derived as Eq. (8) by substituting Eqs. (2) and (3) and the ionization cross section.

$$
\begin{aligned}
K_{i z}= & \frac{1}{n_{e}} \int_{\varepsilon_{i z}}^{\infty} \sigma_{i z}\left(\varepsilon_{e}\right)\left(\frac{2 e \varepsilon_{e}}{m}\right)^{1 / 2} g_{e}\left(\varepsilon_{e}\right) d \varepsilon_{e} \\
= & \alpha \pi\left(\frac{e}{4 \pi \epsilon_{0}}\right)^{2} \frac{g_{x}}{T_{\mathrm{eff}}^{3 / 2}}\left(\frac{2 e}{m}\right)^{1 / 2}\left[\frac{T_{\mathrm{eff}}}{x \varepsilon_{i z} C_{x}^{1 / x}} \Gamma\left(\frac{1}{x}, t_{1}\right)\right. \\
& \left.-\frac{1}{x} \int_{t_{1}}^{\infty} \frac{\exp (-t)}{t} d t\right] .
\end{aligned}
$$

Here, $t_{1}=C_{x}\left(\varepsilon_{i z} / T_{\text {eff }}\right)^{x}$ and $\Gamma(a, x)$ is the upper incomplete Gamma function defined as

$$
\Gamma(a, x)=\int_{x}^{\infty} t^{a-1} \exp (-t) d t .
$$

The integration in the second term of Eq. (8) is the exponential integral of the special functions. Solving Eq. (1) using Eqs. (6) and (8) yields the effective electron temperature $T_{\text {eff }}$ for a given $x$, pressure $\left(n_{g}\right)$, and plasma geometry $(R, l)$.

The EEPFs upstream of the DLs have been experimentally measured in two plasma reactors: the Chi-Kung reactor at the Australian National University [17] and the PMPI reactor at Iwate University [22]. Briefly, Chi-Kung has a $13.7-\mathrm{cm}$-diameter $30-\mathrm{cm}$-long source tube surrounded by a double-saddle field antenna powered from a radio frequency (rf) generator operating at $13.56 \mathrm{MHz}$ and $250 \mathrm{~W}$. The source is contiguously connected to a $30-\mathrm{cm}-$ diameter 30-cm-long aluminum diffusion chamber and an expanding magnetic field of about $130 \mathrm{G}$ in the source is provided by two solenoids. Here the Chi-Kung reactor is operated in argon or xenon and the CFDL forms about $5 \mathrm{~cm}$ upstream of the source exit for a pressure range of 0.2-2 mTorr in argon, and 0.03-0.5 mTorr in xenon [12]. 
The PMPI reactor has a 6.4-cm-diameter 20-cm-long source tube connected to a $26-\mathrm{cm}$-diameter $30-\mathrm{cm}$-long stainless steel diffusion chamber. A double-loop antenna is wound around the source tube $9 \mathrm{~cm}$ upstream of the source exit, and is powered from a rf generator operating at 13.56 MHz and $250 \mathrm{~W}$. In PMPI, the expanding magnetic field of about $100 \mathrm{G}$ in the source is provided by double concentric arrays of permanent magnets. When the PMPI source is operated with an argon pressure of 0.2-1.5 mTorr, the CFDL forms a few centimeters upstream of the source exit [22]. In both experimental systems, the plasmas are terminated at the upstream side by insulator plates, which ensure the current-free condition. Dogleg rf-compensated probes [17] are used to measure the EEPFs at about $8 \mathrm{~cm}$ upstream of the DL in Chi-Kung and at about $3 \mathrm{~cm}$ upstream of the DL in PMPI. The EEPF shown in Fig. 1 was taken in the Chi-Kung reactor and all the EEPFs acquired in the two systems show similar features: a clear change of the slope in logarithm plots of the EEPFs at an energy corresponding to the DL potential drop [17].

The effective electron temperature can be calculated from the experimental EEPF as

$$
\frac{3}{2} T_{\mathrm{eff}}=\frac{1}{n_{e}} \int_{0}^{\infty} \varepsilon_{e} g_{e}\left(\varepsilon_{e}\right) d \varepsilon_{e} .
$$

The effective electron temperature obtained from the EEPF in Fig. 1 measured in Chi-Kung for 0.3 mTorr of argon is about $8.5 \mathrm{eV}$. The theoretical EEPFs with the same effective temperature for various $x$ are calculated using Eq. (2) and plotted in Fig. 1 as dotted $(x=1)$, dashed $(x=1.5)$, solid $(x=2)$, and dot-dashed ( $x=2.5)$ lines, respectively. The experimental EEPF with the low-energy, hightemperature population and the depleted, energetic, low-temperature tail population is found to be well approximated by the theoretical curve for $x=2$, i.e., the Druyvesteyn distribution function.

In order to have confidence that the experimental EEPFs can be fitted by a Druyvesteyn distribution function, an error analysis is performed over the range of pressures used in the experiments. The most appropriate values of $x$ are determined to minimize the error $S(x)$ between the logarithm of the EEPFs $g_{p \text { expt }}\left(\varepsilon_{e}\right)$ in the experiments and $g_{p \text { calc }}\left(\varepsilon_{e}\right)$ calculated using Eq. (2). Here the error $S(x)$ is defined as

$$
S(x)=\int_{T_{\text {eff }}}^{\infty}\left\{\log \left[g_{p \text { expt }}\left(\varepsilon_{e}\right)\right]-\log \left[g_{p \text { calc }}\left(\varepsilon_{e}\right)\right]\right\}^{2} d \varepsilon_{e},
$$

where the integration is carried out above the energy corresponding to the effective electron temperature $T_{\text {eff }}$ since electrons with an energy below $T_{\text {eff }}$ are often lost from the experimental EEPFs [23]. For the calculation of the second term in Eq. (11), i.e., $\log \left[g_{p \text { calc }}\left(\varepsilon_{e}\right)\right]$, the effective electron temperatures $T_{\text {eff }}$ estimated from the experimental EEPFs are used. Figure 2 shows the value of $x$ giving the minimum error $S(x)$ as a function of the

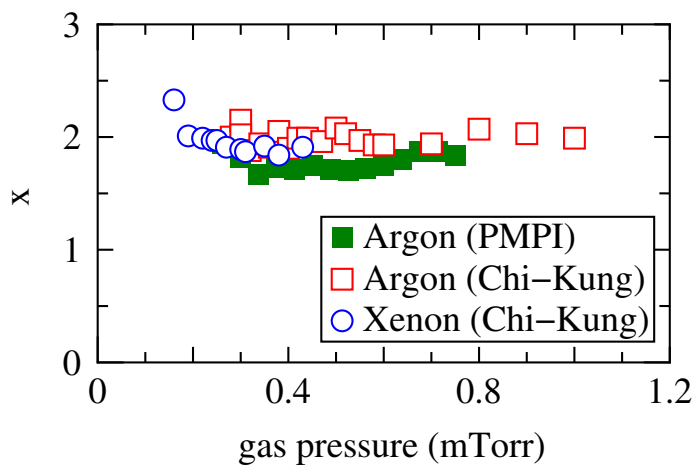

FIG. 2 (color online). The value of $x$ giving the minimum error $S(x)$ defined by Eq. (11).

operating gas pressure for the three experimental data sets: argon in PMPI (filled squares) and argon (open squares) and xenon (open circles) in Chi-Kung. Over this range of gas pressures, the values of $x$ suitable for approximating the experimental EEPFs are close to $x=2$.

Equation (1) is solved for the Druyvesteyn distribution $(x=2)$. The radius $R$ and the length $l$ are selected as the source tube radius (3.2 cm for PMPI and $6.85 \mathrm{~cm}$ for ChiKung) and the axial distance from the upstream insulator plates to the DLs (17 cm for PMPI and $25 \mathrm{~cm}$ for ChiKung), where the ions are assumed to enter the DL and the sheath on the radial and upstream source walls with the Bohm velocity. The theoretical and experimental $T_{\text {eff }}$ are plotted in Fig. 3 for argon in PMPI (crosses with line and filled squares), for argon in Chi-Kung (open triangle with line and open squares), and for xenon in Chi-Kung (open diamonds with line and open circles). The theoretical results are in fairly good agreement with the experimentally obtained effective electron temperatures in the three experimental configurations over the range of investigated gas pressure. Hence, it is demonstrated that the EEPF and EEDF upstream of the CFDL in low-collisional magnetically expanding plasmas can be modeled by the balance equation using Druyvesteyn distribution functions. The dashed line in Fig. 3 shows the analytical $T_{\text {eff }}$ in argon in Chi-Kung geometry in the case of a Maxwellian distribution $(x=1)$ : as expected it shows a significant discrepancy when compared to the experimental data and to the Druyvesteyn analysis. Hence the shape of the EEPF and EEDF, i.e., the value of the parameter $x$, is the critical parameter for determining the effective electron temperature upstream of the low-collisional CFDL.

In some previous reports on the CFDL in magnetically expanding plasmas $[15,24]$, it was assumed that the balance between ionization and particle loss is sustained by an electron beam accelerated by the DL superposed on a Maxwellian distribution function. Here, the present results clearly show that the balance between ionization and particle loss inside the source tube is satisfied by a Druyvesteyn-approximated electron energy distribution with a depleted tail. This CFDL for the Druyvesteyn 


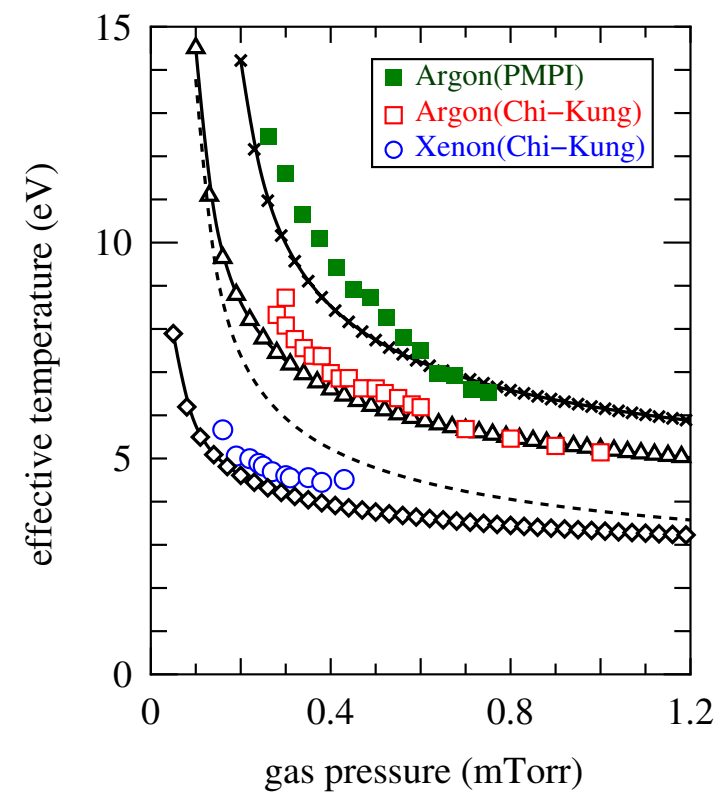

FIG. 3 (color online). Theoretical and experimental effective electron temperatures $T_{\text {eff }}$ in argon in PMPI (crosses with solid line and filled squares), in argon in Chi-Kung (open triangles with solid line and open squares), and in xenon in Chi-Kung (open diamonds with solid lines and open circles) as a function of the gas pressure for a Druyvesteyn distribution $(x=2)$. The dashed line is the analytical results for a Maxwellian distribution $(x=1)$ in argon in Chi-Kung for comparison.

distribution function is not in accordance with the result of Ref. [11] which stipulates the absence of CFDL solutions when there is no hot tail in the electron energy distribution function. This seems to indicate that the low-collisional CFDL is a new class of double layers.

In summary, the electron energy distribution and probability functions (EEDFs and EEPFs) upstream of the CFDL in a low-collisional magnetically expanding plasma have been investigated analytically and experimentally. The energy distribution with a depleted tail above a break energy corresponding to the DL potential drop can be well approximated by a Druyvesteyn distribution. The theoretical effective electron temperatures obtained from the upstream particle balance for the Druyvesteyn distribution agree well with experimental results obtained in two system geometries and for two gases.

This work is partially supported by a Grant-in-Aid for Young Scientists (A) from MEXT, by TEPCO Research
Foundation, and by an Australian Research Council Discovery grant.

*kazunori@iwate-u.ac.jp

[1] B.H. Quon and A. Y. Wong, Phys. Rev. Lett. 37, 1393 (1976).

[2] Ch. Hollenstein, M. Guyot, and E. S. Weibel, Phys. Rev. Lett. 45, 2110 (1980).

[3] N. Sato et al., Phys. Rev. Lett. 46, 1330 (1981).

[4] P. Leung, A. Y. Wong, and B. H. Quon, Phys. Fluids 23, 992 (1980).

[5] N. Hershkowitz et al., Plasma Phys. 23, 903 (1981).

[6] P. Coakley and N. Hershkowitz, Phys. Fluids 22, 1171 (1979).

[7] R. L. Stenzel, M. Ooyama, and Y. Nakamura, Phys. Rev. Lett. 45, 1498 (1980).

[8] F. W. Perkins and Y.C. Sun, Phys. Rev. Lett. 46, 115 (1981).

[9] R. Hatakeyama, Y. Suzuki, and N. Sato, Phys. Rev. Lett. 50, 1203 (1983).

[10] G. Hairapetian and R. L. Stenzel, Phys. Rev. Lett. 61, 1607 (1988).

[11] E. Ahedo and M. Martínez Sánchez, Phys. Rev. Lett. 103, 135002 (2009).

[12] C. Charles, Plasma Sources Sci. Technol. 16, R1 (2007), and references therein.

[13] A. Fredriksen, L. N. Mishra, and H. S. Byhring, Plasma Sources Sci. Technol. 19, 034009 (2010).

[14] R. W. Boswell, E. Marsch, and C. Charles, Astrophys. J. 640, L199 (2006).

[15] M. A. Lieberman and C. Charles, Phys. Rev. Lett. 97, 045003 (2006).

[16] F. F. Chen, Phys. Plasmas 13, 034502 (2006).

[17] K. Takahashi et al., Phys. Plasmas 14, 114503 (2007).

[18] U. Kortshagen, C. Busch, and L.D. Tsendin, Plasma Sources Sci. Technol. 5, 1 (1996).

[19] M. A. Lieberman and A. J. Lichtenberg, Principles of Plasma Discharges and Material Processing (WileyInterscience, Hoboken, NJ, 2005), 2nd ed.

[20] J. T. Gudmundsson, Plasma Sources Sci. Technol. 10, 76 (2001).

[21] H. Amemiya, J. Phys. Soc. Jpn. 66, 1335 (1997).

[22] K. Takahashi and T. Fujiwara, Appl. Phys. Lett. 94, 061502 (2009).

[23] V. A. Godyak, R. B. Piejak, and B. M. Alexandrovich, Plasma Sources Sci. Technol. 1, 36 (1992).

[24] S. C. Thakur et al., Phys. Rev. Lett. 102, 035004 (2009). 